\title{
Adjuvant Hysterectomy for Cervical Cancer Patients Treated with Chemoradiation Therapy: A Systematic Review on the Pathology-Proven Residual Disease Rate
}

\author{
Kim van Kol ${ }^{1,2}$, Renée Ebisch ${ }^{1,3}$, Jurgen Piek ${ }^{3}\left[\right.$, Maaike Beugeling ${ }^{4}$, Tineke Vergeldt $^{3}$ and Ruud Bekkers ${ }^{1,2, *(1)}$ \\ 1 Department of Obstetrics and Gynecology and Catharina Cancer Institute, Catharina Hospital, \\ 5623 EJ Eindhoven, The Netherlands; k.vankol@student.maastrichtuniversity.nl (K.v.K.); \\ renee.ebisch@radboudumc.nl (R.E.) \\ 2 Department of Obstetrics and Gynecology, GROW School for Oncology and Developmental Biology, \\ Maastricht University Medical Center+, 6229 ER Maastricht, The Netherlands \\ 3 Department of Obstetrics and Gynecology, Radboud University Medical Center, 6525 XZ Nijmegen, \\ The Netherlands; jurgen.piek@catharinaziekenhuis.nl (J.P.); tineke.vergeldt@radboudumc.nl (T.V.) \\ 4 Department of Radiation Oncology, Institute Verbeeten (BVI), 5042 SB Tilburg, The Netherlands; \\ beugeling.m@bvi.nl \\ * Correspondence: ruud.bekkers@catharinaziekenhuis.nl
}

check for updates

Citation: van Kol, K.; Ebisch, R.; Piek, J.; Beugeling, M.; Vergeldt, T.; Bekkers, R. Adjuvant Hysterectomy for Cervical Cancer Patients Treated with Chemoradiation Therapy: A Systematic Review on the Pathology-Proven Residual Disease Rate. Cancers 2021, 13, 6190. https:// doi.org/10.3390/cancers13246190

Academic Editor: Massimo Di Maio

Received: 7 November 2021

Accepted: 3 December 2021

Published: 8 December 2021

Publisher's Note: MDPI stays neutral with regard to jurisdictional claims in published maps and institutional affiliations.

Copyright: (c) 2021 by the authors. Licensee MDPI, Basel, Switzerland. This article is an open access article distributed under the terms and conditions of the Creative Commons Attribution (CC BY) license (https:/ / creativecommons.org/licenses/by/ $4.0 /)$.
Simple Summary: The treatment for patients with locally advanced cervical cancer generally consists of platinum-based chemotherapy during external beam radiotherapy, followed by brachytherapy. Some medical centers perform adjuvant hysterectomy after chemoradiation therapy, even though the international guideline advises otherwise. Performing adjuvant hysterectomy after chemoradiation therapy is associated with a high complication rate and the percentage residual disease in adjuvant hysterectomy specimen is unknown. Therefore, the aim of our systematic review was to determine the percentage of residual disease in the adjuvant hysterectomy specimen. Furthermore, we want to determine if there is an association between the time of adjuvant hysterectomy and the percentage residual disease in adjuvant hysterectomy specimens. Findings from this research provide insight into potential complications, survival benefits, and an overtreatment rate. Patients need to be well informed before considering an adjuvant hysterectomy.

Abstract: Objective: To determine the incidence of pathology-proven residual disease in adjuvant hysterectomy specimens in patients with cervical cancer, treated with chemoradiation therapy. Secondly, to assess a possible association for pathology-proven residual disease regarding the time between chemoradiation therapy and adjuvant hysterectomy. Additionally, the survival rate and complication rate were assessed. Methods: PubMed, EMBASE, and the Cochrane database were searched from inception up to 8 March 2021. Results: Of the 4601 screened articles, eleven studies were included. A total of 1205 patients were treated with chemoradiation therapy and adjuvant hysterectomy, ranging from three to twelve weeks after chemoradiation therapy. A total of 411 out of 1205 patients (34\%) had pathology-proven residual disease in the adjuvant hysterectomy specimen. There was no association found in the time between chemoradiation therapy and adjuvant hysterectomy. Follow-up ranged from 2.4 to 245 months, during which 270 patients (22\%) relapsed, and 298 patients (27\%) were deceased. A total of 202 (35\%) complications were registered in 578 patients. Conclusion: there is no association found in the time between chemoradiation therapy and residual disease on adjuvant hysterectomy specimens. The survival rates after chemoradiation therapy and adjuvant hysterectomy are suboptimal, while the risk of complications after adjuvant hysterectomy is high.

Keywords: locally advanced cervical cancer; chemoradiation therapy; adjuvant hysterectomy; complications; survival 


\section{Introduction}

Cervical cancer is the fourth most common malignancy in patients worldwide, within 2018 , an estimated number of 570,000 patients were diagnosed with cervical cancer [1,2]. Approximately $30-40 \%$ of patients with cervical cancer in developed countries have locally advanced cervical cancer at initial diagnosis [3]. Locally advanced cervical cancer is classified as stage IIB-IVA according to the International Federation of Gynecology and Obstetrics (FIGO) 2018 staging system [2].

According to international guidelines, the standard treatment for patients with locally advanced cervical cancer is concurrent chemoradiation therapy. This treatment generally consists of platinum-based chemotherapy during external beam radiotherapy, followed by brachytherapy [4]. There is still a reported overall local pelvic recurrence rate of $14.5 \%$ after treatment with chemoradiation therapy for patients with stage IIB-IVA disease [5,6]. Reported 5-year overall survival rates range from $83 \%$ for stage IB to $32 \%$ for stage IVA [7]. Even though the international guidelines advise otherwise, some medical centers do perform adjuvant hysterectomy after chemoradiation therapy. Adjuvant hysterectomy after chemoradiation therapy has not been shown to be effective in improving survival outcomes, but it seems to reduce the risk of local recurrence [8,9].

If a patient has received chemoradiation therapy, the morphologic response continues after chemoradiation therapy. Therefore, the total benefit of chemoradiation therapy is achieved for several weeks after the last radiation treatment [10]. Medical centers performing routine adjuvant hysterectomy are inconsistent in the time between chemoradiation therapy and adjuvant surgery. However, the time between chemoradiation therapy and adjuvant hysterectomy has not been assessed before [4,9]. Performing adjuvant hysterectomy after chemoradiation therapy is associated with a high complication risk because of radiation-induced tissue damage and reduced propensity to healing [11,12]. This may negatively influence the quality of life of patients.

The aim of this review is to determine the incidence of pathology-proven residual disease in adjuvant hysterectomy specimens in patients treated with chemoradiation therapy because of cervical cancer. Furthermore, we aimed to assess a possible association with time between chemoradiation therapy and adjuvant hysterectomy. Secondary outcome measures were survival rate and complications after an adjuvant hysterectomy.

\section{Materials and Methods}

\subsection{Protocol and Registration}

A protocol was designed according to the Preferred Reporting Items for Systematic Reviews and Meta-Analyses (PRISMA) guideline and registered in PROSPERO (registration number CRD42020196399). In this systematic review, adjuvant hysterectomy was defined as planned surgery after primary treatment with chemoradiation therapy, without determining the presence or absence of residual disease. Adequate chemoradiation therapy is defined as a total dose radiation therapy of at least 70 Gy (external beam radiation therapy and brachytherapy). Preferable, with daily external beam radiotherapy total dose of 45 Gy with concomitant platinum-based chemotherapy (recommended weekly cisplatin $40 \mathrm{mg} / \mathrm{m}^{2}$ for $5-6$ cycles) followed by brachytherapy.

\subsection{Literature Search}

PubMed, EMBASE and Cochrane library were searched for articles published from inception up to 8 March 2021. The selection criteria combined synonyms for cervix uteri, cervical cancer, chemoradiation therapy, hysterectomy and adjuvant hysterectomy and included MeSH terms (Supporting Information S1). Duplicate articles were manually filtered using the bibliographic database Endnote X9.

\subsection{Eligibility Criteria}

All titles and abstracts were independently assessed by two researchers (KvK, RE) and any discrepancies were resolved by a third researcher (TV). The selection of articles 
was independently assessed for full text by two reviewers. Studies describing patients with cervical cancer, requiring chemoradiation therapy, and treated with adjuvant hysterectomy were suitable for inclusion. In this systematic review, there were no restrictions regarding language and the different FIGO-classifications. The time between chemoradiation therapy and adjuvant hysterectomy, the pathology results after adjuvant hysterectomy, and survival rates had to be described in the study. Reviews of literature, case reports and case series with five patients or less, conference abstracts and letters to the editor were excluded. Articles describing patients treated with salvage hysterectomy and studies treating patients with inadequate chemoradiation therapy were not included. Studies were included when $>90 \%$ of patients were treated according to our adequate chemoradiation therapy definition. For studies updating prior published series, the most recent data were retained. To reduce the risk of including duplicate patients in the systematic review, articles written by the same authors and/or university were assessed by three reviewers (KvK, RE, TV), based on year, inclusion period, number of patients, disease stage, chemoradiation therapy regimen, surgical treatment, and the follow-up period. From coinciding articles, the articles with the most recent information, the most included patients, the broadest stage range, and the largest follow-up period were included.

\subsection{Data Collection}

From the relevant articles, the following information was extracted: inclusion period, country, number of patients treated with chemoradiation therapy, number of patients with adjuvant hysterectomy, FIGO stage, histology, dose and regimen of chemoradiation therapy, dose of brachytherapy, type of adjuvant hysterectomy, time until adjuvant hysterectomy, pathology results after surgery, complications after adjuvant hysterectomy, recurrence rate and survival outcome. An association between CRT and adjuvant hysterectomy was considered when the percentage of positive pathology diminished in time. The overall survival rate was calculated by dividing the number of deaths by the total of people treated with chemoradiation therapy and adjuvant hysterectomy. The complication rate was calculated by dividing the number of reported complications by the number of patients treated with chemoradiation therapy and adjuvant hysterectomy.

\subsection{Assessment of Risk of Bias}

Two reviewers $(\mathrm{KvK}, \mathrm{RE})$ independently determined the quality of the included articles according to the Newcastle-Ottawa Scale for cohort studies. The quality assessment was based on three categories: selection, comparability, and outcomes. A total of nine stars could be awarded, a study with six or more stars was defined as a high-quality study, between three and five stars was defined as fair quality and less than three stars as poor quality.

\section{Results}

The search revealed 4601 articles after systematical removal of duplicates. After screening title and abstract, 80 studies were evaluated for full text. After reading full text, 69 studies did not fulfill the search query, 32 studies were excluded based on the same cohorts of patients, and 27 studies were excluded because of inadequate chemoradiation therapy. Ten studies were excluded because of incomplete patient information (time till adjuvant hysterectomy, pathology results, follow-up, recurrence, or survival rate were missing). No additional studies were identified by checking the reference list. Figure 1 shows the flow diagram of the selection process. 


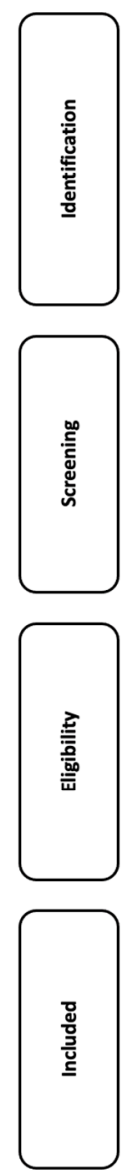

Articles identified through PubMed ( $n=1588)$

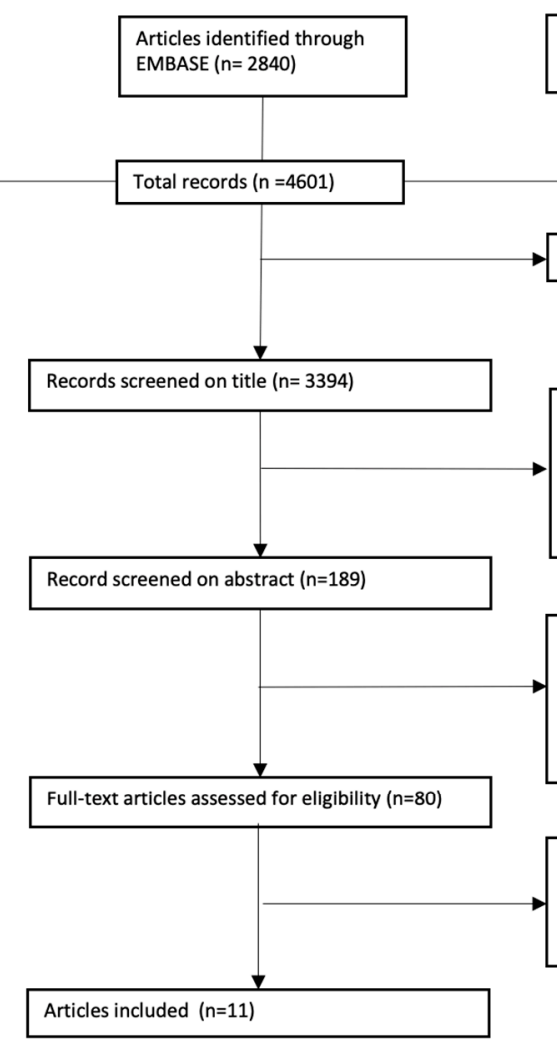

Articles identified through Cochrane library $(n=173)$

PubMed (n=1

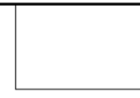

Duplicates removed $(n=1207)$

xcluded ( $n=3205$ ) Other treatment: 754

Case report: 420

Review: 278

Surgical technique: 40

Record screened on abstract $(n=189)$

Excluded ( $\mathrm{n}=109)$

Conference abstract: 49

Different subject: 18

Other treatment: 27

Language: 7

Early-stage cervical cancer:

Surgical technique: 1

Review: 1

Case report: 1

Letter: 1

Duplicate patients: 32

Inadequate CRT: 278

Incomplete patient information: 10

Figure 1. Flowchart of the selection procedure.

\subsection{Characteristics of Included Studies}

The total of eleven included articles consisted of six retrospective cohort studies, four prospective cohort studies and one phase III randomized controlled trial. Results of the included studies [13-23] are summarized in Table 1.

\subsection{Newcastle-Ottawa Scale}

Of the total group of eleven studies, ten studies were assessed as good or fair quality and one study was assessed as poor quality according to the Newcastle-Ottawa Scale for cohort studies (Table 2). 


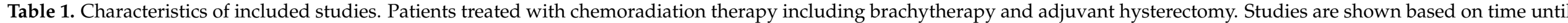
adjuvant hysterectomy.

\begin{tabular}{|c|c|c|c|c|c|c|c|c|c|c|c|c|c|}
\hline $\begin{array}{l}\text { Author } \\
\text { (Year) }\end{array}$ & $\begin{array}{l}\text { Inclusion } \\
\text { Period } \\
\text { (Years) }\end{array}$ & $\begin{array}{c}\text { Total Number of } \\
\text { Patients (Treated } \\
\text { with } \\
\text { Chemoradiation } \\
\text { Therapy and } \\
\text { Adjuvant } \\
\text { Hysterectomy) }\end{array}$ & $\begin{array}{l}\text { Age Me- } \\
\text { dian/Mean } \\
\text { (Range) }\end{array}$ & FIGO Stage *1 & Histology & $\begin{array}{l}\text { Chemoradiation } \\
\text { Therapy regimen }\end{array}$ & $\begin{array}{l}\text { Brachytherapy } \\
\text { Dosage }\end{array}$ & $\begin{array}{l}\text { Type of Adjuvant } \\
\text { Hysterectomy }\end{array}$ & $\begin{array}{l}\text { Time } \\
\text { Until } \\
\text { Adju- } \\
\text { vant } \\
\text { Hys- } \\
\text { terec- } \\
\text { tomy }\end{array}$ & $\begin{array}{c}\text { Positive } \\
\text { Pathol- } \\
\text { ogy } \\
\text { Results } \\
\text { After } \\
\text { Surgery } \\
n(\%)\end{array}$ & $\begin{array}{l}\text { Follow-up } \\
\text { Period Me- } \\
\text { dian/Mean } \\
\text { (Range) }\end{array}$ & $\begin{array}{l}\text { Recurr- } \\
\text { ence } n \\
(\%)\end{array}$ & Survival $n(\%)$ \\
\hline $\begin{array}{c}\text { Keys } \\
(1999) \\
{[13]}\end{array}$ & $\begin{array}{l}1992- \\
1997\end{array}$ & 374 (183) & Unknown & $\mathrm{IB}=183$ & $\begin{array}{c}\mathrm{SCC}=147 \\
\mathrm{AC}=9 \\
\mathrm{ASC}=17 \\
\text { Other }=10\end{array}$ & $\begin{array}{l}45 \text { Gy EBRT with } \\
\text { concomitant } \\
\text { cisplatin }(40 \\
\left.\mathrm{mg} / \mathrm{m}^{2}\right)^{* 2}\end{array}$ & 30 Gy & $\begin{array}{c}\text { Extrafascial } \\
\text { hysterectomy *3 }\end{array}$ & $\begin{array}{c}3-6 \\
\text { weeks }\end{array}$ & $88(48 \%)$ & $\begin{array}{l}\text { Median } 26 \\
\text { months }\end{array}$ & $38(21 \%)$ & $27 \operatorname{died}(15 \%)$ \\
\hline $\begin{array}{l}\text { Eitan } \\
\text { (2010) } \\
{[14]}\end{array}$ & $\begin{array}{l}2003- \\
2006\end{array}$ & 23 & $\begin{array}{l}\text { Median } 50 \\
\text { year (range, } \\
30-67 \text { year) }\end{array}$ & $\mathrm{IB} 2=20, \mathrm{IIA}=3$ & $\begin{aligned} \mathrm{SCC} & =22, \\
\mathrm{AC} & =1\end{aligned}$ & $\begin{array}{l}45 \text { Gy EBRT with } \\
\text { concomitant } \\
\text { cisplatin }(35 \\
\left.\mathrm{mg} / \mathrm{m}^{2}\right)^{* 4}\end{array}$ & $\underset{\mathrm{Gy})}{14 \mathrm{~Gy}(2 \times 7}$ & $\begin{array}{l}\text { Simple extra-fascial } \\
\text { total abdominal } \\
\text { hysterectomy and } \\
\text { bilateral salpingo- } \\
\text { oophorectomy (and } \\
\text { in } 16 \text { patients } \\
\text { PLND). }\end{array}$ & $\begin{array}{l}\text { Median } \\
5 \text { weeks, } \\
\text { range } \\
14-52 \\
\text { days. }\end{array}$ & $11(48 \%)$ & $\begin{array}{l}\text { Median } 20 \\
\text { months } \\
\text { (range, 10-50 } \\
\text { months) }\end{array}$ & $4(17.4 \%)$ & $2(8.7 \%)$ \\
\hline $\begin{array}{c}\text { Colombo } \\
\text { (2009) } \\
{[15]}\end{array}$ & $\begin{array}{l}2000- \\
2008\end{array}$ & 102 & $\begin{array}{l}\text { Mean } 44 \\
\text { year (range, } \\
24-74 \text { year) }\end{array}$ & $\begin{array}{c}\mathrm{IB}=28, \mathrm{IIA}=13 \\
\mathrm{IIB}=61\end{array}$ & $\begin{array}{c}\mathrm{SCC}=91 \\
\mathrm{AC}=10 \\
\text { other }=1\end{array}$ & $\begin{array}{l}45 \text { Gy EBRT with } \\
\text { concomitant } \\
\text { cisplatin }(40 \\
\mathrm{mg} / \mathrm{m}^{2}\end{array}$ & $15 \mathrm{~Gy} * 5$ & $\begin{array}{l}56 \text { abdominal radical } \\
\text { hysterectomy and } 46 \\
\text { total laparoscopic } \\
\text { radical hysterectomy }\end{array}$ & $\begin{array}{c}4-6 \\
\text { weeks }\end{array}$ & $36(35 \%)$ & $\begin{array}{l}\text { Mean } 31.2 \\
\text { months }\end{array}$ & $\begin{array}{c}32(31.4 \%): \\
18(17.6 \%) \\
\text { local and } \\
14(13.7 \%) \\
\text { distant }\end{array}$ & $\begin{array}{l}\text { 3-year OS } 82 \% \text {, } \\
19 \text { died }(18.6 \%)\end{array}$ \\
\hline $\begin{array}{c}\text { Fröbe } \\
\text { (2014) } \\
{[16]}\end{array}$ & $\begin{array}{l}2002- \\
2008\end{array}$ & 24 & $\begin{array}{l}\text { Median } 50 \\
\text { year (range, } \\
39-71 \text { year) }\end{array}$ & $\begin{array}{c}\mathrm{IB}=8 \\
\mathrm{IIA}=3, \mathrm{IIB}=13\end{array}$ & $\begin{aligned} \mathrm{SCC} & =19 \\
\mathrm{AC} & =5\end{aligned}$ & $\begin{array}{l}40 \text { Gy EBRT with } \\
\text { concomitant } \\
\text { cisplatin }(30 \\
\left.\mathrm{mg} / \mathrm{m}^{2}\right)^{* 6}\end{array}$ & $\underset{\mathrm{Gy})}{28 \mathrm{~Gy}(4 \times 7}$ & $\begin{array}{c}\text { Radical } \\
\text { hysterectomy and } \\
\text { bilateral salphingo- } \\
\text { oophorectomy } \\
\text { without lymph node } \\
\text { dissection }\end{array}$ & 6 weeks & $12(50 \%)$ & $\begin{array}{l}\text { Median } 67 \\
\text { months } \\
\text { (range 4-107 } \\
\text { months) }\end{array}$ & $\begin{array}{l}\text { three } \\
\text { distant } \\
\text { metastases } \\
(12.5 \%)\end{array}$ & $\begin{array}{l}\text { Six died }(25 \%) \\
\text { three without } \\
\text { evidence of } \\
\text { disease }\end{array}$ \\
\hline $\begin{array}{c}\text { Cheewakric } \\
(2005) \\
{[17]}\end{array}$ & $\begin{array}{c}\text { ngkrajig- } \\
2001\end{array}$ & 34 & $\begin{array}{l}\text { Mean } 44 \\
\text { year (range, } \\
\text { 30-66 year) }\end{array}$ & $\begin{array}{c}\mathrm{IB} 1=4, \mathrm{IB} 2=25 \\
\mathrm{IIA}=5\end{array}$ & $\begin{array}{c}\mathrm{SCC}=22 \\
\mathrm{AC}=11 \\
\mathrm{ASC}=1\end{array}$ & $\begin{array}{l}\text { 46-50 Gy ERBT } \\
\text { with weekly } \\
\text { concomitant } \\
\text { cisplatin }(40 \\
\left.\mathrm{mg} / \mathrm{m}^{2}\right)\end{array}$ & $\begin{array}{c}30 \text { Gy }(4 \times 7.5 \\
\text { Gy })\end{array}$ & $\begin{array}{l}\text { Extra-fascial } \\
\text { hysterectomy }\end{array}$ & 6 weeks & $16(47 \%)$ & $\begin{array}{l}\text { Median } 42 \\
\text { months } \\
\text { (range 7-58 } \\
\text { months) }\end{array}$ & $\begin{array}{l}\text { Six } \\
\text { (18.2\%):one } \\
\text { distant, } \\
\text { three local } \\
\text { and two } \\
\text { combined } \\
\end{array}$ & $\begin{array}{l}\text { five deaths } \\
(14.7 \%)\end{array}$ \\
\hline $\begin{array}{l}\text { Cagetti } \\
(2020) \\
{[18]}\end{array}$ & $\begin{array}{l}2012- \\
2017\end{array}$ & $145(90)$ & $\begin{array}{l}\text { Median } 54 \\
\text { year (range, } \\
24-90 \text { year) }\end{array}$ & $\begin{array}{c}\mathrm{IB}=33, \mathrm{IIA}=9 \\
\mathrm{IIB}=40 \\
\mathrm{III}-\mathrm{IV}=8\end{array}$ & $\begin{array}{l}\mathrm{SCC}=66 \\
\mathrm{AC}=19 \\
\text { other }=5\end{array}$ & $\begin{array}{l}45 \text { Gy EBRT with } \\
\text { concomitant } \\
\text { cisplatin }(40 \\
\left.\mathrm{mg} / \mathrm{m}^{2}\right)^{* 7}\end{array}$ & $\begin{array}{c}27.5 \text { Gy }(5 \times 5.5 \\
\text { Gy) }\end{array}$ & $\begin{array}{c}\text { Radical } \\
\text { hysterectomy }\end{array}$ & $\begin{array}{c}5-8 \\
\text { weeks } \\
\text { median: } \\
61 \pm 26 \\
\text { days }\end{array}$ & $28(31 \%)$ & $\begin{array}{l}\text { Median } 30.8 \\
\text { months } \\
\text { (range, } \\
26.0-30.0 \\
\text { months) }\end{array}$ & $\begin{array}{l}11 \text { local } \\
(12.2 \%)\end{array}$ & $\begin{array}{c}\text { 3-year OS } \\
50-90 \% \\
\text { depending on } \\
\text { pathology } \\
\text { results }\end{array}$ \\
\hline $\begin{array}{c}\text { Bigsby } \\
(2011) \\
{[19]}\end{array}$ & $\begin{array}{l}1999- \\
2009\end{array}$ & 69 & $\begin{array}{l}\text { Mean } 47 \\
\text { year (range, } \\
27-82 \text { year) }\end{array}$ & IB2 = 69 & $\begin{array}{l}\mathrm{SCC}=55 \mathrm{AC} \\
=11 \mathrm{ASC}=3\end{array}$ & $\begin{array}{c}45-50.4 \text { Gy EBRT } \\
\text { with concomitant } \\
\text { cisplatin }(25-40 \\
\left.\mathrm{mg} / \mathrm{m}^{2}\right)^{* 8}\end{array}$ & $\begin{array}{c}15-18 \text { Gy }(3 \times \\
5-6 \mathrm{~Gy})\end{array}$ & $\begin{array}{l}\text { Extra-fascial total } \\
\text { abdominal } \\
\text { hysterectomy with } \\
\text { common and } \\
\text { para-aortic } \\
\text { lymphadenectomy }\end{array}$ & $\begin{array}{c}\text { 6-8 } \\
\text { weeks }\end{array}$ & $35(51 \%)$ & $\begin{array}{l}\text { Mean } 61.7 \\
\text { months } \\
\text { (range } \\
10.9-122.5 \\
\text { months) }\end{array}$ & $\begin{array}{l}16(23.2 \%) \\
2 \text { local, } 14 \\
\text { distant }\end{array}$ & $\begin{array}{l}16 \text { deaths } \\
(23.2 \%) \text { three } \\
\text { were of } \\
\text { unrelated } \\
\text { causes. }\end{array}$ \\
\hline
\end{tabular}


Table 1. Cont.

\begin{tabular}{|c|c|c|c|c|c|c|c|c|c|c|c|c|c|}
\hline $\begin{array}{l}\text { Author } \\
\text { (Year) }\end{array}$ & $\begin{array}{l}\text { Inclusion } \\
\text { Period } \\
\text { (Years) }\end{array}$ & $\begin{array}{c}\text { Total Number of } \\
\text { Patients (Treated } \\
\text { with } \\
\text { Chemoradiation } \\
\text { Therapy and } \\
\text { Adjuvant } \\
\text { Hysterectomy) }\end{array}$ & $\begin{array}{l}\text { Age Me- } \\
\text { dian/Mean } \\
\text { (Range) }\end{array}$ & FIGO Stage ${ }^{* 1}$ & Histology & $\begin{array}{l}\text { Chemoradiation } \\
\text { Therapy regimen }\end{array}$ & $\begin{array}{l}\text { Brachytherapy } \\
\text { Dosage }\end{array}$ & $\begin{array}{l}\text { Type of Adjuvant } \\
\text { Hysterectomy }\end{array}$ & $\begin{array}{l}\text { Time } \\
\text { Until } \\
\text { Adju- } \\
\text { vant } \\
\text { Hys- } \\
\text { terec- } \\
\text { tomy }\end{array}$ & $\begin{array}{c}\text { Positive } \\
\text { Pathol- } \\
\text { ogy } \\
\text { Results } \\
\text { After } \\
\text { Surgery } \\
n(\%)\end{array}$ & $\begin{array}{l}\text { Follow-up } \\
\text { Period Me- } \\
\text { dian/Mean } \\
\text { (Range) }\end{array}$ & $\begin{array}{l}\text { Recurr- } \\
\text { ence } n \\
(\%)\end{array}$ & Survival $n(\%)$ \\
\hline $\begin{array}{c}\text { Kunos } \\
(2010) \\
{[20]}\end{array}$ & Unknown & $464(175)$ & $\begin{array}{l}\text { Median } \\
40 \text { year } \\
\text { (range, } \\
\text { 21-81 year) }\end{array}$ & $\mathrm{IB}=175$ & $\begin{array}{c}\mathrm{SCC}=140, \\
\mathrm{AC}=9, \\
\mathrm{ACS}=16, \\
\text { Other }=10\end{array}$ & $\begin{array}{l}45 \text { Gy EBRT with } \\
\text { concomitant } \\
\text { cisplatin } \\
\left(40 \mathrm{mg} / \mathrm{m}^{2}\right)\end{array}$ & $30 \mathrm{~Gy}$ & $\begin{array}{l}\text { Total extrafascial. } \\
\text { hysterectomy }\end{array}$ & $\begin{array}{c}\text { 6-8 } \\
\text { weeks }\end{array}$ & $35 \underset{* 9}{(20 \%)}$ & $\begin{array}{l}\text { Median } 128 \\
\text { months }\end{array}$ & $\begin{array}{c}39(22.3 \%): \\
16(9 \%) \\
\text { local, } 23 \\
(13 \%) \\
\text { distant }\end{array}$ & $\begin{array}{l}30 \text { cancer } \\
\text { related deaths, } \\
15 \text { death of } \\
\text { unrelated cause. }\end{array}$ \\
\hline $\begin{array}{c}\text { Zheng } \\
(2017) \\
{[21]}\end{array}$ & $\begin{array}{l}2008- \\
2013\end{array}$ & 314 (163) & $\begin{array}{l}\text { Median } 51 \\
\text { (range, } \\
26-73 \text { year) }\end{array}$ & $\begin{array}{c}\mathrm{IB} 2=35, \mathrm{IIA}= \\
71, \mathrm{IIB}=57\end{array}$ & $\begin{aligned} \mathrm{SCC} & =136, \\
\mathrm{AC} & =27\end{aligned}$ & $\begin{array}{c}46-50 \text { Gy EBRT } \\
\text { with concomitant } \\
\text { cisplatin } \\
\left(40 \mathrm{mg} / \mathrm{m}^{2}\right)^{* 10}\end{array}$ & 25-30 Gy & $\begin{array}{c}\text { Radical } \\
\text { hysterectomy and } \\
\text { PLND }\end{array}$ & $\begin{array}{l}\text { Within } 8 \\
\text { weeks }\end{array}$ & $\begin{array}{c}65 \\
(39.9 \%)\end{array}$ & Unknown & $\begin{array}{l}48(29.4 \%) \\
12 \text { local, } 29 \\
\text { metastases, } \\
\text { seven local } \\
\text { and distant }\end{array}$ & $\begin{array}{c}\text { 3-year OS } 87.1 \% \\
21 \text { deaths } \\
(12.9 \%)\end{array}$ \\
\hline $\begin{array}{c}\text { Touboul } \\
(2009) \\
{[22]}\end{array}$ & $\begin{array}{l}1998- \\
2007\end{array}$ & 150 & $\begin{array}{l}\text { Median } 47 \\
\text { year (range, } \\
19-77 \text { year) }\end{array}$ & $\begin{array}{c}\mathrm{IB} 2=48, \mathrm{II}=91 \\
\mathrm{III}=10, \mathrm{IV}=1\end{array}$ & $\begin{array}{c}\text { SCC }=108 \\
\text { AC }=26 \\
\text { other }=16\end{array}$ & $\begin{array}{l}45 \text { Gy EBRT with } \\
\text { concomitant } \\
\text { cisplatin } \\
\left(40 \mathrm{mg} / \mathrm{m}^{2}\right)^{* 11}\end{array}$ & $15 \mathrm{~Gy}$ & $\begin{array}{c}\text { Radical } \\
\text { hysterectomy } \\
(n=44) \text { or simple } \\
\text { extra fascial } \\
\text { hysterectomy }(n= \\
\text { 106) with or without } \\
\text { para-aortic and /or } \\
\text { PLND }\end{array}$ & $\begin{array}{c}8-10 \\
\text { weeks }\end{array}$ & $78(52 \%)$ & $\begin{array}{l}\text { Median } 43.2 \\
\text { months } \\
\text { (range, } \\
2.4-127.2 \\
\text { months) }\end{array}$ & $41(27 \%)$ & $\begin{array}{l}37 \text { deaths } \\
(24.7 \%)\end{array}$ \\
\hline Total & & 2077(1205) & $\begin{array}{c}\text { Range, } \\
19-90 \text { years }\end{array}$ & $\begin{array}{c}\text { IB = 427, } \\
\text { IB1 = 4, IB2 = } \\
197, \text { II = 91, } \\
\text { IIA }=104, \text { IIB }= \\
261, \text { III = 111, IV } \\
=1 \text { IVA }=1 \\
\text { III-IVA }=8\end{array}$ & $\begin{array}{l}\mathrm{SCC}=955 \\
\mathrm{AC}=158 \\
\mathrm{ASC}=37 \\
\text { Other }=55\end{array}$ & & & & & $411(34 \%)$ & $\begin{array}{l}\text { Range } \\
2.4-245 \\
\text { months }\end{array}$ & $\begin{array}{l}270 \text { lo- } \\
\mathrm{cal} / \text { distant }\end{array}$ & $\begin{array}{l}223 \text { died based } \\
\text { on } 10 \text { studies of } \\
\text { which } 21 \text { died } \\
\text { of unrelated } \\
\text { causes to } \\
\text { cervical cancer. }\end{array}$ \\
\hline
\end{tabular}

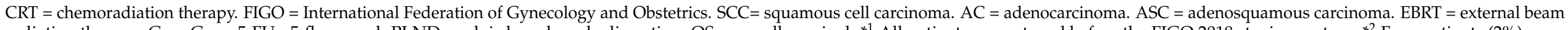

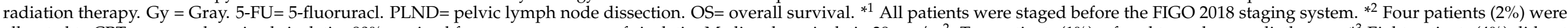

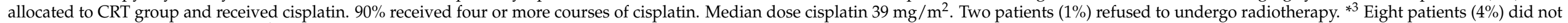

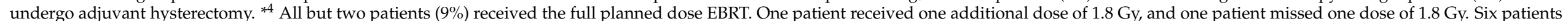

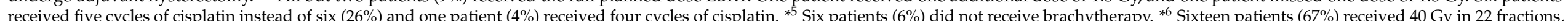

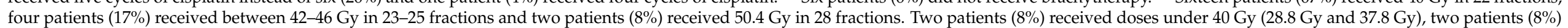

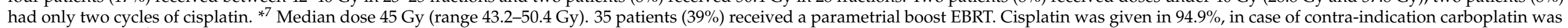

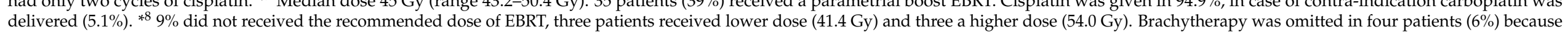

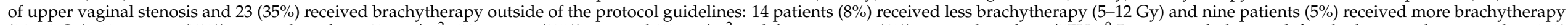

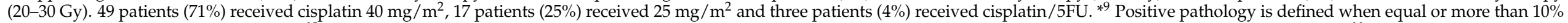

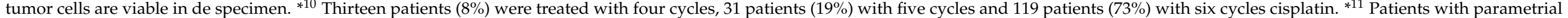
spread and/or bulky pelvic nodes on imaging received a pelvic lateral boost of 10-15 Gy, unknown how much patients received this boost. 
Table 2. Newcastle Ottawa scale for cohort studies.

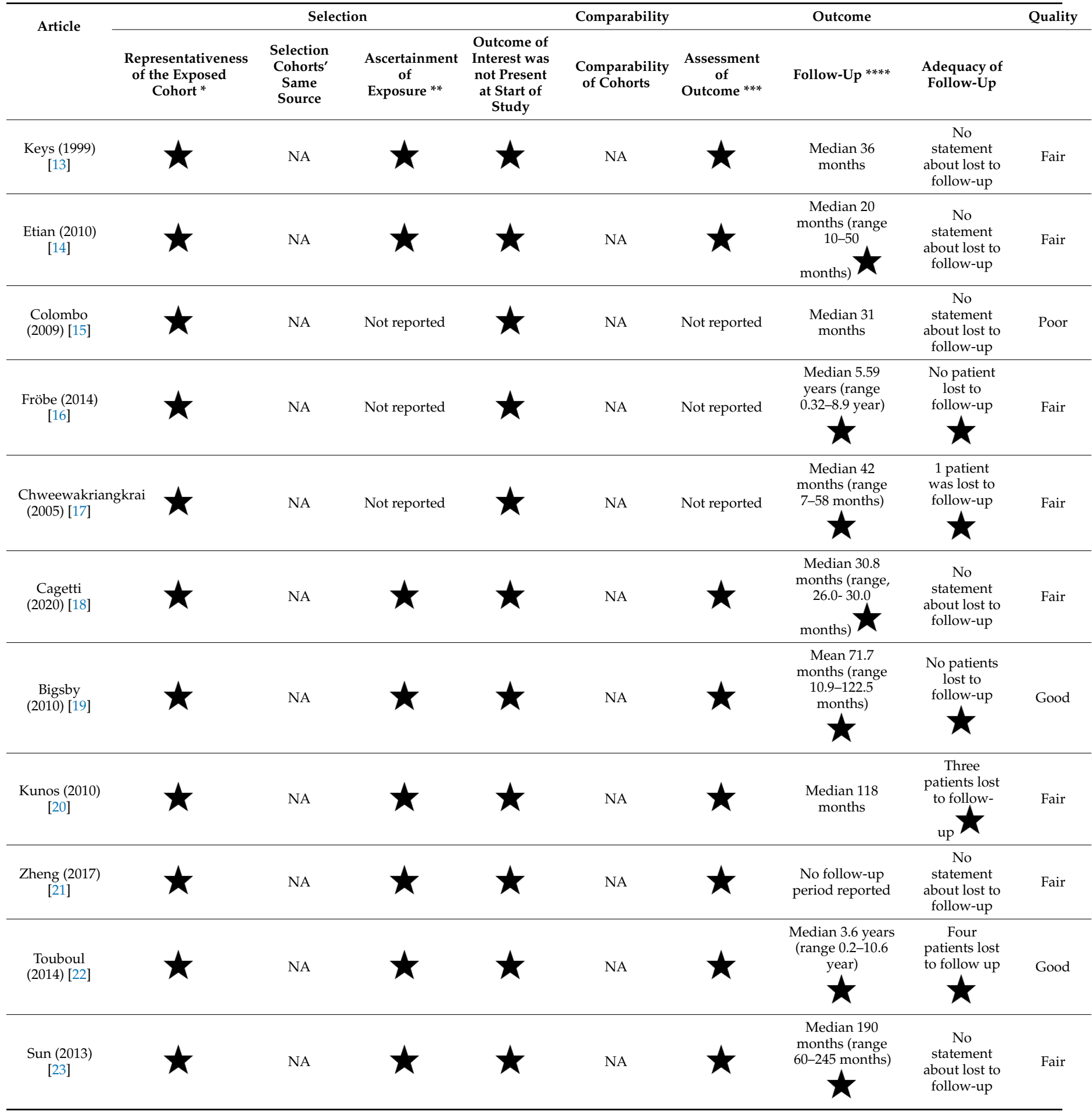

${ }^{*}$ Representativeness of the exposed cohort: all included studies representative for women with residual cervical cancer after chemoradiation therapy treated with salvage surgery. ${ }^{* *}$ Ascertainment of exposure: all with database or medical records. ${ }^{* * *}$ Assessment of outcome: all with medical records. ${ }^{* * * *}$ Follow-up period $\geq 12$ months was assessed as long enough for outcomes to occur. $\star$ : fulfilled the criteria of the Newcastle Ottawa Scale for this item. 


\subsection{Analysis}

Eleven studies were included, representing a total of 2077 patients of which 1205 patients $(58 \%)$ were treated with chemoradiation therapy and adjuvant hysterectomy. Patients were included with an age range of 19-90 years. Of this group, $955(79 \%)$ patients were diagnosed with squamous cell carcinoma, 158 (13\%) with adenocarcinoma, $37(3 \%)$ with adenosquamous carcinoma and $55(5 \%)$ with another histological subtype. Adjuvant hysterectomy was performed between three and twelve weeks after chemoradiation therapy. Residual disease in the pathology specimen was found in $34 \%$ of the patients. All studies performed adjuvant hysterectomy at different time points after chemoradiation therapy. No association was found for pathology-proven residual disease regarding the time between chemoradiation therapy and adjuvant hysterectomy (Figure 2).

\section{Residual disease in pathology specimen after chemoradiation therapy and adjuvant hysterectomy}

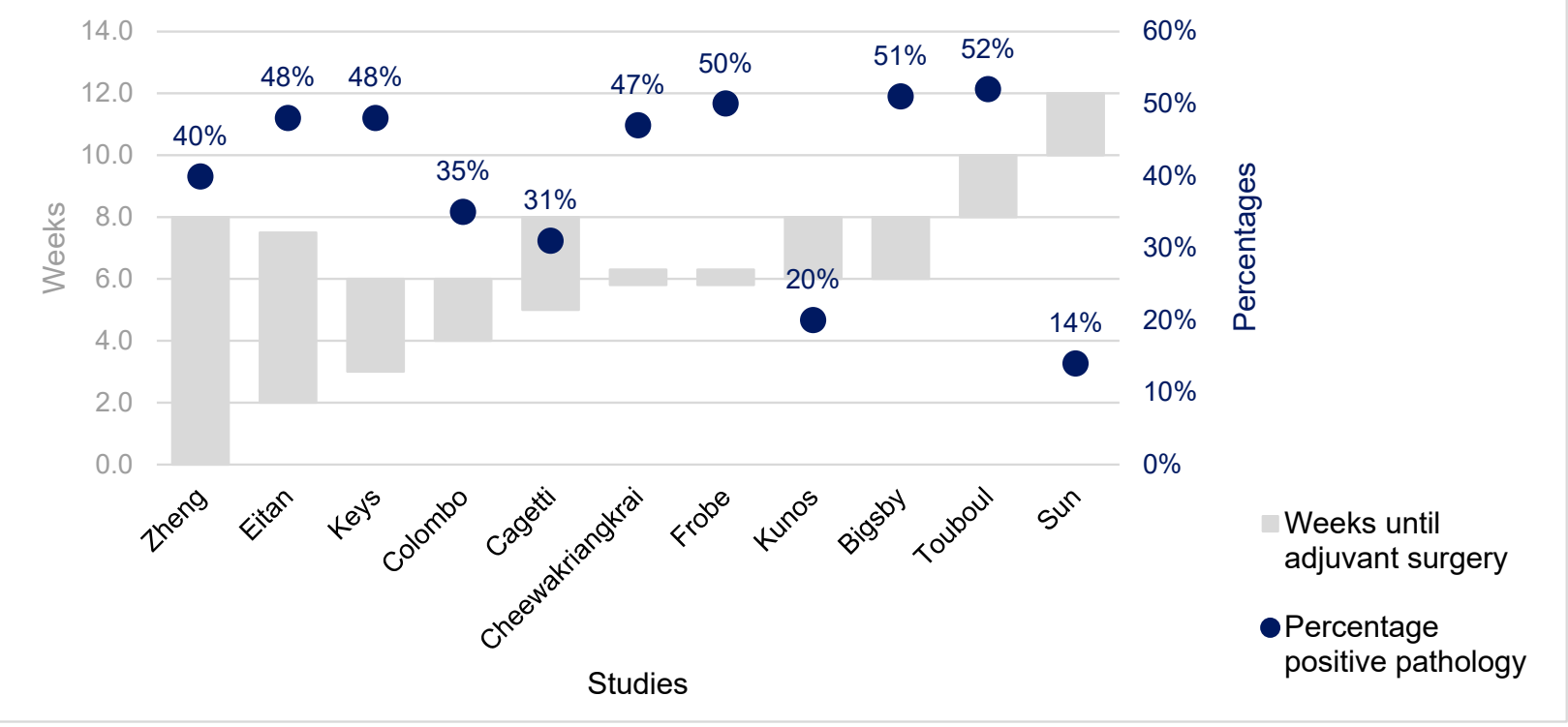

Figure 2. Percentage pathology-proven residual disease after adequate chemoradiation therapy and adjuvant hysterectomy.

Recurrence of disease after treatment with chemoradiation therapy and adjuvant hysterectomy was observed in 270 patients (22\%). Recurrence was pathology-proven in the study of Sun et al. In all other studies the way of determining the recurrence of disease is unknown. The survival rate is based on ten studies and 1115 patients, of which 298 patients $(27 \%)$ deceased because of cervical cancer and 28 patients $(3 \%)$ deceased because of causes unrelated to cervical cancer. The follow-up period is described in ten studies and the patients were followed during a range of 2.4 and 245 months (Table 1).

A total of 202 complications related to adjuvant hysterectomy were registered in 578 patients treated with chemoradiation therapy and adjuvant hysterectomy $(35 \%)$. A total of 27 fistulas (5\%) was reported, of which sixteen fistulas to the urinary tract and eleven fistulas to the gastro-intestinal tract. A total of 53 complications of the urinary tract was reported $(9 \%)$ five injuries to the urinary tract and five ureteral stenoses. Six complications to the female genital tract were reported $(1 \%)$, two patients with vaginal necrosis and two with vaginal stenosis. Eighteen gastro-intestinal complications were reported (3\%) of which two intestinal injuries and a total of thirteen infections was reported $(2 \%)$ of which seven with an abscess, four with peritonitis and two patients with pelvic infections. Other complications were reporter 81 times, of which four deaths related to postoperative morbidity, four ruptures of the iliac vessel during surgery and one pulmonary embolism (Table 3). 
Table 3. Complications after chemoradiation therapy including brachytherapy followed by adjuvant hysterectomy.

\begin{tabular}{|c|c|c|c|c|c|c|c|c|}
\hline $\begin{array}{l}\text { Auteur } \\
\text { (Year) }\end{array}$ & $\begin{array}{l}\text { Patients } \\
\text { Treated } \\
\text { with } \\
\text { Adjuvant } \\
\text { Hysterec- } \\
\text { tomy }\end{array}$ & Fistula & $\begin{array}{l}\text { Urinary } \\
\text { Tract }\end{array}$ & $\begin{array}{c}\text { Female } \\
\text { Genital } \\
\text { Tract }\end{array}$ & Gastrointestin & 1 Infection & Other & Total \\
\hline $\begin{array}{c}\text { Eitan } \\
(2010) \\
{[14]}\end{array}$ & 23 & $\begin{array}{l}\text { One recto- } \\
\text { vaginal } \\
\text { fistula }\end{array}$ & 10 cystitis & & 11 diarrheas & & $\begin{array}{l}\text { Seven anemia, two } \\
\text { lymphedema }\end{array}$ & 31 \\
\hline $\begin{array}{c}\text { Colombo } \\
(2009) \\
{[15]}\end{array}$ & 144 & $\begin{array}{l}\text { Seven } \\
\text { urinary } \\
\text { fistulas, } \\
\text { two } \\
\text { digestive } \\
\text { fistulas }\end{array}$ & $\begin{array}{l}15 \text { vesical } \\
\text { dysfunc- } \\
\text { tion, } 12 \\
\text { cystitis, } \\
\text { three } \\
\text { bladder } \\
\text { injury, two } \\
\text { ureteral } \\
\text { injury }\end{array}$ & & $\begin{array}{c}\text { Two } \\
\text { digestive } \\
\text { injury }\end{array}$ & $\begin{array}{l}\text { Two pelvic } \\
\text { infections }\end{array}$ & $\begin{array}{l}\text { Six intraoperative } \\
\text { hemorrhages, five } \\
\text { other, two } \\
\text { symptomatic } \\
\text { lymphocysts, one } \\
\text { pulmonary } \\
\text { embolism, one } \\
\text { postoperative } \\
\text { hemorrhage }\end{array}$ & 64 \\
\hline $\begin{array}{c}\text { Bigsby } \\
(2011) \\
{[19]}\end{array}$ & 69 & $\begin{array}{l}\text { One entero- } \\
\text { cutaneous } \\
\text { fistula, one } \\
\text { rectovagi- } \\
\text { nal } \\
\text { fistula }\end{array}$ & $\begin{array}{c}\text { One } \\
\text { ureteral } \\
\text { stenosis } \\
\text { requiring } \\
\text { stent, one } \\
\text { cystotomy } \\
\text { with repair }\end{array}$ & $\begin{array}{c}\text { Two } \\
\text { vaginal } \\
\text { stenosis, } \\
\text { one vaginal } \\
\text { fault necro- } \\
\text { sis/grade } \\
\text { four } \\
\text { proctitis, } \\
\text { one vaginal } \\
\text { vault } \\
\text { necrosis, } \\
\end{array}$ & $\begin{array}{c}\text { one complete } \\
\text { small bowel } \\
\text { obstruction }\end{array}$ & & $\begin{array}{l}\text { One deep venous } \\
\text { thrombosis }\end{array}$ & 10 \\
\hline $\begin{array}{c}\text { Touboul } \\
(2009) \\
{[22]}\end{array}$ & 150 & $\begin{array}{c}\text { Five } \\
\text { ureteral } \\
\text { fistula, five } \\
\text { bowel } \\
\text { fistulas, } \\
\text { one } \\
\text { bladder } \\
\text { fistula }\end{array}$ & $\begin{array}{c}\text { Two } \\
\text { ureteral } \\
\text { stenosis, } \\
\text { two } \\
\text { bladder } \\
\text { retention, } \\
\text { one urinary } \\
\text { inconti- } \\
\text { nence }\end{array}$ & $\begin{array}{c}\text { One } \\
\text { vaginal } \\
\text { vault } \\
\text { dehiscence } \\
\text { with } \\
\text { abscess }\end{array}$ & $\begin{array}{c}\text { Two bowel } \\
\text { obstruction, } \\
\text { one } \\
\text { epigastralgia }\end{array}$ & $\begin{array}{c}\text { Five } \\
\text { abscesses, } \\
\text { three } \\
\text { peritonitis }\end{array}$ & $\begin{array}{c}\text { Nine } \\
\text { lymphedemas, } \\
\text { eight lymphocysts, } \\
\text { three chylous } \\
\text { ascites, three } \\
\text { phlebitis, two } \\
\text { deaths related to } \\
\text { postoperative } \\
\text { morbidity, two } \\
\text { rupture of iliac } \\
\text { vessels, two } \\
\text { wound dehiscence }\end{array}$ & 57 \\
\hline $\begin{array}{l}\text { Sun } \\
(2013) \\
{[23]}\end{array}$ & 192 & $\begin{array}{c}\text { Two } \\
\text { ureteral } \\
\text { fistula, one } \\
\text { bowel } \\
\text { fistula, one } \\
\text { bladder } \\
\text { fistula }\end{array}$ & $\begin{array}{c}\text { Two } \\
\text { ureteral } \\
\text { stenosis, } \\
\text { one } \\
\text { bladder } \\
\text { retention, } \\
\text { one urinary } \\
\text { inconti- } \\
\text { nence }\end{array}$ & $\begin{array}{c}\text { One } \\
\text { vaginal } \\
\text { vault } \\
\text { dehiscence } \\
\text { with } \\
\text { abscess }\end{array}$ & $\begin{array}{l}\text { One bowel } \\
\text { obstruction }\end{array}$ & $\begin{array}{c}\text { Two } \\
\text { abscesses, } \\
\text { one } \\
\text { peritonitis }\end{array}$ & $\begin{array}{c}15 \text { lymphocysts, } \\
\text { three } \\
\text { lymphedemas, two } \\
\text { death related to } \\
\text { postoperative } \\
\text { morbidity, two } \\
\text { chylous ascites, } \\
\text { two ruptures of } \\
\text { iliac vessels, two } \\
\text { wound dehiscence, } \\
\text { one phlebitis }\end{array}$ & 40 \\
\hline Total & 578 & 27 & 53 & 6 & 18 & 13 & 81 & 202 \\
\hline
\end{tabular}




\section{Discussion}

This systematic review shows a mean pathology-proven residual disease in 34\% (range, $14-52 \%$ ) of all patients undergoing adjuvant hysterectomy after chemoradiation therapy for cervical cancer. No association was found between the time between chemoradiation therapy and for pathology-proven residual disease on adjuvant hysterectomy specimens. The survival rate after chemoradiation therapy and adjuvant hysterectomy seem suboptimal, and adjuvant hysterectomy has a high risk of complications, including death.

\subsection{Comparison with Existing Literature}

In this systematic review, the percentage of residual disease on adjuvant hysterectomy specimens did not diminish in time. In the included studies, adjuvant hysterectomy was performed within twelve weeks after chemoradiation therapy. However, we believe it might be plausible that the incidence of positive pathology could decrease because the morphologic response sometimes continues for several weeks after the last treatment of chemoradiation therapy [10]. In addition to this hypothesis, Eifel et al. reported $22 \%$ local recurrences for patients with stage III cervical cancer after treatment with only chemoradiation therapy [5]. Which is lower than the mean 34\% residual disease in the surgical specimen in our review. This may indicate that when (salvage) surgery is performed too early after chemoradiation therapy patients are overtreated. Thereby, two previously published studies assessed residual disease detection by biopsy. The study of Boers et al. shows that residual disease detected by biopsy eight to ten weeks after chemoradiation therapy is a poor prognostic factor to identify patients with residual disease who may be salvaged by surgery [24]. The study by Hoeijmakers et al. implies that a biopsy to prove residual disease should be taken twelve until sixteen weeks after completing chemoradiation therapy, to select patients for salvage surgery [25]. These studies indicate that detection of residual disease should not take place early after chemoradiation therapy.

As previously mentioned, two recent meta-analyses determined the survival rates for adjuvant hysterectomy after chemoradiation. They concluded that adjuvant hysterectomy seemed to reduce the risk of local recurrence [8,9]. One limitation of these studies is that the results are based on only two randomized controlled trials [26,27]. The review of literature in our study shows an overall recurrence rate (distant and local recurrence) of $22 \%$ after adjuvant hysterectomy and chemoradiation therapy. Previous studies reported an overall recurrence rate after treatment with only chemoradiation therapy of up to $37 \%[5,6]$. We reported an overall survival of $73 \%$ after chemoradiation therapy and adjuvant hysterectomy, which seems to be equivalent to treatment with chemoradiation therapy alone. Previous studies reported 5-year overall survival rates for patients with stage IB-IVA ranges between $32 \%$ and $83 \%$ [7]. A hypothesis for the decreased recurrence rate after adjuvant hysterectomy and chemoradiation therapy, compared to chemoradiation therapy alone, is that patients with residual disease after chemoradiation therapy are directly treated with salvage surgery. Patients with residual disease after chemoradiation therapy are an unfavorable group of patients, which are likely to relapse. However, standard adjuvant hysterectomy after chemoradiation therapy harbors the risk that the majority of patients are unnecessary treated and thus exposed to the (post)operative risks. Besides, more recent studies use a higher total dose of radiation therapy, which results in lower reported local recurrence rates. For example, the retroEMBRACE study used a mean D90 (EQD210) for high and intermediate risk clinical target volumes of $87 \pm 15$ Gy and $69 \pm 8$ Gy respectively. They reported $9.6 \%$ of local failures, of which $6.4 \%$ had true local recurrences [7]. This indicates even less reason to perform an adjuvant hysterectomy.

Additionally, adjuvant hysterectomy after chemoradiation therapy is associated with a high complication risk. The risk of complications after adjuvant hysterectomy is higher in comparison with primary surgery because adjuvant hysterectomy takes place in a previously irradiated area with the unpredictable healing quality of the tissue [28]. In our systematic review adjuvant hysterectomy is associated with a percentage of major complications of $35 \%$. The mortality rate after adjuvant hysterectomy in our systematic 
review is $0.7 \%$. The study of Magrina et al. reported a mortality rate of $0.5 \%$ after primary radical hysterectomy [29]. A major complication with high implications for patients is the development of a fistula. Fistulas can occur due to irradiation, surgery, or a combination of both [30]. The study of Hilton et al. reported an overall fistula rate after a hysterectomy of $0.13 \%$ and a fistula rate of $1 \%$ after radical hysterectomy for patients with cervical cancer [31]. In this review the studies reported an overall fistula rate of $5 \%$, this implicates that the chance of developing fistulas increases after the combination of chemoradiation therapy and adjuvant hysterectomy. Most of the complications after adjuvant hysterectomy turn out to be unnecessary because $66 \%$ of the patients were overtreated with adjuvant hysterectomy. Patients should be clearly informed on the survival benefits and the disadvantages like complications and the over-treatment rate of non-routinely adjuvant hysterectomy. To reduce the number of patients needed to harm by adjuvant hysterectomy, less invasive procedures, such as radiological imaging or biopsies, should be performed first, to determine the presence of residual disease before performing surgery. However, the best period to determine residual disease by radiological imaging, biopsy, as well as performing adjuvant or salvage surgery, should be further investigated.

\subsection{Strengths and Weaknesses}

One of the strengths of this study is that the dose and regimen of chemoradiation therapy that patients received were assessed by a radiation oncologist, this ensures that all patients were treated via a comparable chemoradiation therapy treatment schedule, similar to high income countries. However, the evaluation of data is not without limitations, there is much heterogenicity among the included studies. First, the time to complete chemoradiation therapy was not an inclusion criterion, which could influence survival outcomes. Second, not all studies performed the same type of adjuvant hysterectomy after chemoradiation therapy. Differing between simple hysterectomy and extended hysterectomy with or without lymph node dissection, and between open versus laparoscopic surgery. This could influence the complication rate. Analyzing the different surgery types regarding recurrence, survival and complications was not possible because of missing data. Third, the preoperative doses of chemoradiation therapy given to patients were nearly always less than the planned dose. Fourth, pathological residual disease rate can be underestimated because the viable microscopic residual disease can be missed in surgical specimens. Fifth, it was not possible to calculate the 2-, 3- or 5-years overall survival rate as the data was not representative, which could overestimate or underestimate the survival rate.

\section{Conclusions}

The majority of patients treated with adjuvant hysterectomy as a routine procedure are over-treated. No association was found between the time between chemoradiation therapy and pathology-proven residual disease on adjuvant hysterectomy specimens. The survival rate after adjuvant hysterectomy seems suboptimal, and the risk of major complications after adjuvant hysterectomy is high. To prevent high overtreatment rates by adjuvant hysterectomy it should be considered to use less invasive procedures to determine the presence of residual disease before performing surgery after chemoradiation therapy. Patients need to be well informed on potential complications, survival benefits, and a potential overtreatment rate before considering an adjuvant hysterectomy.

Supplementary Materials: The following are available online at https:/ / www.mdpi.com/article/10 $.3390 /$ cancers13246190/s1, supporting information S1: Literature search

Author Contributions: Conceptualization, R.B.; Data curation K.v.K., R.E. and T.V.; formal analysis K.v.K., R.E. and M.B.; writing-original draft K.v.K., Writing—review \& editing R.E., J.P., M.B., T.V. and R.B. All authors have read and agreed to the published version of the manuscript.

Funding: This research received no external funding.

Conflicts of Interest: The authors declare no conflict of interest. 


\section{References}

1. WHO. Cervical Cancer 2019. Available online: https://www.who.int/cancer/prevention/diagnosis-screening/cervical-cancer/ en/ (accessed on 2 December 2020).

2. Bhatla, N.; Aoki, D.; Sharma, D.N.; Sankaranarayanan, R. Cancer of the cervix uteri. Int. J. Gynecol. Obstet. 2018, 143, 22-36. [CrossRef] [PubMed]

3. Cancer Research UK. Cancer Statistics for the UK. Available online: https://www.cancerresearchuk.org/health-professional/ cancer-statistics-for-the-uk (accessed on 14 January 2020).

4. Cibula, D.; Pötter, R.; Planchamp, F.; Avall-Lundqvist, E.; Fischerova, D.; Haie Meder, C.; Köhler, C.; Landoni, F.; Lax, S.; Lindegaard, J.C.; et al. The European Society of Gynaecological Oncology/European Society for Radiotherapy and Oncology/European Society of Pathology Guidelines for the Management of Patients With Cervical Cancer. Int. J. Gynecol. Cancer 2018, 472, 641-655. [CrossRef] [PubMed]

5. Eifel, P.J.; Winter, K.; Morris, M.; Levenback, C.; Grigsby, P.W.; Cooper, J.; Rotman, M.; Gershenson, D.; Mutch, D.G. Pelvic irradiation with concurrent chemotherapy versus pelvic and para-aortic irradiation for high-risk cervical cancer: An update of Radiation Therapy Oncology Group Trial (RTOG) 90-01. J. Clin. Oncol. 2004, 22, 872-880. [CrossRef] [PubMed]

6. Pötter, R.; Dimopoulos, J.; Georg, P.; Lang, S.; Waldhäusl, C.; Wachter-Gerstner, N.; Weitmann, H.; Reinthaller, A.; Knocke, T.H.; Wachter, S.K.C. Clinical impact of MRI assisted dose volume adaptation and dose escalation in brachytherapy of locally advanced cervix cancer. Radiother. Oncol. 2007, 83, 148-155. [CrossRef] [PubMed]

7. Sturdza, A.; Pötter, R.; Fokdal, L.U.; Haie-Meder, C.; Tan, L.T.; Mazeron, R.; Petric, P.; Šegedin, B.; Jurgenliemk-Schulz, I.M.; Nomden, C.; et al. Image guided brachytherapy in locally advanced cervical cancer: Improved pelvic control and survival in RetroEMBRACE, a multicenter cohort study. Radiother. Oncol. 2016, 120, 428-433. [CrossRef]

8. Shi, D.; Liang, Z.; Zhang, C.; Zhang, H.L.X. The effect of surgery on the survival status of patients with locally advanced cervical cancer after radiotherapy/chemoradiotherapy: A meta-analysis. BMC Cancer 2018, 8, 308. [CrossRef] [PubMed]

9. Shim, S.H.; Kim, S.N.; Chae, S.H.; Kim, J.E.L.S. Impact of adjuvant hysterectomy on prognosis in patients with locally advanced cervical cancer treated with concurrent chemoradiotherapy: A meta-analysis. J. Gynecol. Oncol. 2018, 29, e25. [CrossRef]

10. Baskar, R.; Lee, K.A.; Yeo, R.; Yeoh, K.W. Cancer and radiation therapy: Current advances and future directions. Int. J. Med. Sci. 2012, 9, 193-199. [CrossRef]

11. Kol, K.G.G.; Ebisch, R.M.F.; Piek, J.M.J.; Zusterzeel, P.L.M.; Vergeldt, T.F.M.; Bekkers, R.L.M. Salvage surgery for patients with residual disease after chemoradiation therapy for locally advanced cervical cancer: A systematic review on indication, complications, and survival. Acta Obs. Gynecol. Scand. 2021, 100, 1176-1185. [CrossRef] [PubMed]

12. Viswanathan, A.N.; Lee, L.J.; Eswara, J.R.; Horowitz, N.S.; Konstantinopoulos, P.A.; Mirabeau-Beale, K.L.; Rose, B.S.; Von Keudell, A.G.; Wo, J.Y. Complications of pelvic radiation in patients treated for gynecologic malignancies. Cancer 2014, 120, $3870-3883$. [CrossRef]

13. Keys, H.M.; Bundy, B.N.; Stehman, F.B.; Muderspach, L.I.; Chafe, W.E.; Suggs, C.L.; Walker, J.L.; Gersell, D.; Mackey, D. Cisplatin, radiation, and adjuvant hysterectomy compared with radiation and adjuvant hysterectomy for bulky stage IB cervical carcinoma. N. Engl. J. Med. 1999, 340, 1154-1161. [CrossRef]

14. Eitan, R.; Levavi, H.; Peled, Y.; Brenner, R.; Sabah, G.; Ben-Arie, A.; Dgani, R.; Fishman, A.; Sulkes, A.; Fenig, E.; et al. Should simple hysterectomy be added after chemo-radiation for stage IB2 and bulky IIA cervical carcinoma? Aust. N. Z. J. Obs. Gynaecol. 2010, 50, 289-293. [CrossRef]

15. Colombo, P.E.; Bertrand, M.M.; Gutowski, M.; Mourregot, A.; Fabbro, M.; Saint-Aubert, B.; Quenet, F.; Gourgou, S.; Kerr, C.; Rouanet, P. Total laparoscopic radical hysterectomy for locally advanced cervical carcinoma (stages IIB, IIA and bulky stages IB) after concurrent chemoradiation therapy: Surgical morbidity and oncological results. Gynecol. Oncol. 2009, 114, 404-409. [CrossRef]

16. Fröbe, A.; Jones, G.; Bokulić, T.; Mrčela, I.; Budanec, M.; Murgić, J.; Jakšić, B.; Prpić, M.; Bolanča, A.; Kusić, Z. High-doserate brachytherapy and concurrent chemoradiotherapy followed by surgery for stage Ib-Ilb cervical cancer: Single institution experience. Anticancer Res. 2014, 34, 3861-3866. [PubMed]

17. Cheewakriangkrai, C.; Srisomboon, J.; Chitapanarux, I.; Suprasert, P.; Phongnarisorn, C.; Sitthicha Siriaree, K.C. Concurrent cisplatin-based chemoradiation and adjuvant hysterectomy for bulky stage IB-IIA cervical cancer. J. Med. Assoc. Thai. 2005, 88, 1331-1337.

18. Cagetti, L.; Zemmour, C.; Minsat, M.; Lambaudie, E.; Houvenaeghel, G.; Provansal, M.; Cappiello, M.A.; Rua, S.; Jauffret, C.; Ferré, M.; et al. Lessons from radiochemotherapy and modern image-guided adaptive brachytherapy followed by hysterectomy. Gynecol. Oncol. 2020, 156, 328-334. [CrossRef]

19. Bigsby, G.E.; Robert, W.; Holloway Ahmad, S.; Michael, D.; Sombeck, G.E. Chemoradiation with adjuvant hysterectomy for stage IB-2 cervical cancer: A 10-year experience. Gynecol. Surg. 2012, 9, 327-333. [CrossRef]

20. Kunos, C.; Ali, S.; Abdul-Karim, F.W.; Stehman, F.B.; Waggoner, S. Posttherapy residual disease associates with long-term survival after chemoradiation for bulky stage 1B cervical carcinoma: A Gynecologic Oncology Group study. Am. J. Obs. Gynecol. 2010, 203, 351.e1-351.e8. [CrossRef]

21. Zheng, D.; Mou, H.P.; Diao, P.; Li, X.M.; Zhang, C.L.; Jiang, J.; Chen, J.L.; Wang, L.S.; Wang, Q.; Zhou, G.Y.; et al. Chemoradiotherapy in combination with radical surgery is associated with better outcome in cervical cancer patients. Oncotarget 2018, 9, 2866-2875. [CrossRef] 
22. Touboul, C.; Uzan, C.; Mauguen, A.; Gouy, S.; Rey, A.; Pautier, P.; Lhommé, C.; Duvillard, P.; Haie-Meder, C.; Morice, P. Prognostic Factors and Morbidities After Completion Surgery in Patients Undergoing Initial Chemoradiation Therapy for Locally Advanced Cervical Cancer. Oncologist 2010, 15, 405-415. [CrossRef]

23. Sun, L.; Sheng, X.; Jiang, J.; Li, X.; Liu, N.; Liu, Y.; Zhang, T.; Li, D.; Zhang, X.; Wei, P. Surgical morbidity and oncologic results after concurrent chemoradiation therapy for advanced cervical cancer. Int. J. Gynecol. Obstet. 2014, 125, 111-115. [CrossRef]

24. Boers, A.; Arts, H.J.G.; Klip, H.; Nijhuis, E.R.; Pras, E.; Hollema, H.; Wisman, G.B.A.; Nijman, H.W.; Mourits, M.J.E.; Reyners, A.K.L.; et al. Radical surgery in patients with residual disease after (chemo)radiation for cervical cancer. Int. J. Gynecol. Cancer 2014, 24, 1276-1285. [CrossRef]

25. Hoeijmakers, Y.M.; Snyers, A.; van Ham, M.A.P.C.; Zusterzeel, P.L.M.; Bekkers, R.L.M. Cervical biopsy after chemoradiation for locally advanced cervical cancer to identify residual disease: A retrospective cohort study. J. Surg. Oncol. 2019, 2, 2-6.

26. Morice, P.; Rouanet, P.; Rey, A.; Romestaing, P.; Houvenaeghel, G.; Boulanger, J.C.; Leveque, J.; Cowen, D.; Mathevet, P.; Malhaire, J.P.; et al. NoResults of the GYNECO 02 study, an FNCLCC phase III trial comparing hysterectomy with no hysterectomy in patients with a (clinical and radiological) complete response after chemoradiation therapy for stage IB2 or II cervical cancer. Oncologist 2012, 17, 64-71. [CrossRef] [PubMed]

27. Cetina, L.; Garcia-Arias, A.; Candelaria, M.; Cantú, D.; Rivera, L.; Coronel, J.; Bazan-Perkins, B.; Flores, V.; Gonzalez, A.D.G.A. Brachytherapy versus radical hysterectomy after external beam chemoradiation: A non-randomized matched comparison in IB2-IIB cervical cancer patients. World J. Surg. Oncol. 2009, 7, 19. [CrossRef]

28. Wydra, D.; Emerich, J.; Sawicki, S.; Ciach, K.; Marciniak, A. Major complications following exenteration in cases of pelvic malignancy: A 10-year experience. World J. Gastroenterol. 2006, 12, 1115-1119. [CrossRef]

29. Magrina, J.F.; Goodrich, M.A.; Weaver, A.L.; Podratz, K.C. Modified radical hysterectomy: Morbidity and mortality. Gynecol. Oncol. 1995, 59, 277-282. [CrossRef]

30. Narayanan, P.; Nobbenhuis, M.; Reynolds, K.M.; Sahdev, A.; Reznek, R.H.; Rockall, A.G. Fistulas in malignant gynecologic disease: Etiology, imaging, and management. Radiographics 2009, 29, 1073-1083. [CrossRef]

31. Hilton, P.; Cromwell, D.A. The risk of vesicovaginal and urethrovaginal fistula after hysterectomy performed in the English National Health Service-a retrospective cohort study examining patterns of care between 2000 and 2008. BJOG An. Int. J. Obs. Gynaecol. 2012, 119, 1447-1454. [CrossRef] [PubMed] 